\title{
IMPACTO DE LA CRISIS ECONÓMICA Y LOS CAMBIOS EN LAS POLÍTICAS SOCIALES EN EL PERFIL, NECESIDADES Y PRESTACIONES DE LAS PERSONAS USUARIAS DE LOS SERVICIOS SOCIALES MUNICIPALES. ANÁLISIS DE CASO LOCAL EN LA REGIÓN DE MURCIA (ESPAÑA)
}

\author{
Economic crisis impact and social policy changes in the profile, needs \\ and benefits of municipal social services' users. A local case study in the \\ region of Murcia (Spain)
}

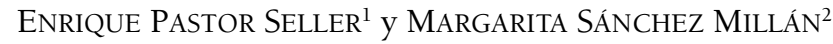

\section{Resumen}

El artículo presenta los resultados de una investigación empírica orientada a conocer y analizar el impacto de la crisis económica y los cambios normativos en las necesidades y prestaciones de las personas usuarias del sistema de servicios sociales de atención primaria. Para su consecución se ha realizado una investigación longitudinal de necesidades y prestaciones en un municipio de la Comarca Oriental de la Región de Murcia durante el período comprendido entre 1996 y 2011, comparando sus resultados con investigaciones similares de ámbito regional y estatal. Así mismo se presenta un análisis transversal (2012) y de tendencias. La metodología empleada se ha basado en la diversidad de técnicas y fuentes de recolección y análisis de datos a partir del análisis del universo de personas usuarias, concretamente 4.562 personas y 3.325 unidades familiares, lo que proporciona a la investigación unos resultados fiables, contrastables y transferibles. Los resultados obtenidos permiten verificar la hipótesis de partida de que los cambios en el perfil de las personas usuarias, las necesidades y los servicios / recursos aplicados por la estructura básica de los servicios sociales municipales están directamente relacionados con las dinámicas de la crisis social y económica y el desarrollo de las políticas/normativas de bienestar social relacionadas directa e indirectamente con los servicios sociales.

Palabras clave: crisis, servicios sociales, políticas sociales, necesidades sociales, bienestar social.

\begin{abstract}
This article presents the results of empirical research with the objective of discovering and analyzing the impact of the economic crisis and regulatory changes in needs and benefits of the primary care social services system users. To this end, a longitudinal study on the needs and benefits in a municipality in the eastern region of Murcia have been conducted from 1996 to 2011, comparing their results with similar regional and state investigations. It also presents a cross-sectional and trends analysis (2012). The methodology used is based on a diversity of techniques, pickup sources and data analysis of the users' universe, specifically 4,562 individuals and 3,325 households, thereby providing a transferable and contrastable reliable research result. The obtained results verify the hypothesis that changes in the profile of the users, needs and services/resources applied by the basic structure of the municipal social services is directly related to the dynamics of social and economic crisis and policy development/welfare regulations related directly and indirectly to social services.
\end{abstract}

Key words: crisis, social services, social policies, social needs, social welfare.

\begin{tabular}{lll}
\hline Recibido: 21/10/2013 & Aceptado: 07/11/2013 & Publicado: 03/12/2013 \\
\hline
\end{tabular}

1. Profesor Titular de Universidad. Decano de la Facultad de Trabajo Social de la Universidad de Murcia. IP Grupo de Investigación Trabajo Social y Servicios Sociales. epastor@um.es

2. Graduada en Trabajo Social y Responsable Servicios Sociales de Beniel (Murcia). 


\section{Introducción}

El Sistema Público de Servicios Sociales en España lo conforman el conjunto de servicios y prestaciones cuya finalidad es la promoción del desarrollo pleno y libre de la persona dentro de la sociedad para la obtención de un mayor bienestar social y mejor calidad de vida, así como la prevención y eliminación de las causas que conducen a la marginación social; estando integrado por todas las estructuras y servicios públicos. Un sistema basado en los principios de universalidad, responsabilidad pública, igualdad, equidad, prevención y dimensión comunitaria, promoción de la autonomía personal, atención personalizada e integral, inserción y normalización, proximidad, solidaridad, calidad, participación y cooperación y colaboración (Ministerio de Sanidad, Servicios Sociales e Igualdad, 2013). Por consiguiente, sus elementos de identidad son: ámbito específico de necesidades sociales de referencia (derechos sociales que garantiza); oferta específica y estructurada de prestaciones sociales; red de equipamientos; normativa, planificación y presupuestos propios.

Son numerosas las definiciones existentes respecto de los servicios sociales de atención primaria, por las distintas leyes autonómicas y autores/as. Sin ser el objeto de este artículo entrar en una disertación al respecto, podemos partir de entenderlos como aquellas estructuras y equipamientos básicos del Sistema Público de Servicios Sociales, diseñados sobre espacios poblacionales dimensionados que garantizan las Prestaciones Básicas del Sistema a los ciudadanos, mediante atenciones de carácter individual, familiar y/o colectivo, a través de recursos propios o como cauce de acceso a otros Servicios Sociales o de Bienestar Social. Se organizan territorialmente por medio de los Centros de Servicios Sociales y unidades zonales, los cuales tienen un carácter universal y polivalente en el ámbito más próximo al ciudadano (ámbito local) y constituyen la estructura básica.

El sistema encuentra su primera referencia en un ámbito específico de necesidades sociales a las que debe dar respuesta: información y orientación sobre el acceso a recursos; convivencia personal y familiar; integración social; cobertura de necesidades básicas y participación social. Para su atención se dota de recursos o prestaciones técnicas, económicas y materiales para la gestión de las prestaciones básicas de información y orientación, apoyo a la unidad convivencial y ayuda a domicilio; alojamiento alternativo, prevención e inserción social y fomento de la solidaridad social y la cooperación social.

La Constitución, a pesar de la indefinición del término servicios sociales, otorga a las Comunidades Autónomas las competencias en materia de servicios sociales, las cuales son asumidas en las Leyes Orgánicas de los Estatutos de las diferentes Comunidades Autónomas y desarrolladas a través de 
las Leyes de Servicios Sociales y de otras vinculadas con la protección social. A partir de 2006 y como consecuencia, fundamentalmente, de la promulgación de la Ley 39/2006, de 14 de diciembre, de Promoción de la Autonomía Personal y Atención a las personas en situación de Dependencia ${ }^{3}$, surgen las denominadas leyes de servicios sociales de «tercera generación $»^{4}$ caracterizadas, entre otras, por: el derecho subjetivo a las prestaciones y servicios; la ampliación y consolidación de derechos y deberes de las personas usuarias; la importancia de la calidad; la introducción del derecho subjetivo y la elaboración de catálogos / carteras de las prestaciones y servicios (Casado, 2010; Pastor 2012a; Vila, 2012). La mencionada Ley ha sufrido recientemente modificaciones ${ }^{5}$ sustanciales $^{6}$ repercutiendo de manera desfavorable en los derechos y prestaciones inicialmente regulados (Ramírez, 2012, García, Barriga, Ramírez y Santos, 2013).

La Ley 7/1985, de 2 de abril, Reguladora de las Bases del Régimen Local ${ }^{7}$, actualmente en proceso de revisión en razón del proyecto de Ley aprobado por el Consejo de Ministros del 22 de febrero de 2013, establece la competencia de los municipios en la prestación de los servicios sociales (arts. $25.2 \mathrm{~K}$

3. Publicada en B.O.E. núm.: 299 de 15 de diciembre de 2006.

4. Ley Foral 15/2006, de 14 de diciembre, de servicios sociales B.O.E. núm.: 27 de 31 de enero. Ley de Cantabria 2/2007, de 27 de marzo de Derechos y Servicios Sociales B.O.C. núm.: 66 de 3 de abril. Ley 12/2007, de 11 de octubre de Cataluña B.O.E. núm.: 266 de 6 de noviembre. Ley 13/2008, de 3 de diciembre, de servicios sociales de Galicia D.O.G. núm.: 245 de 18 de diciembre. Ley 12/2008, de 5 de diciembre, de servicios sociales del País Vasco B.O.P.V. núm: 246 de 24 de diciembre. Ley 4/2009, de 11 de junio, de servicios sociales de las Illes Baleares B.O.B.B núm.: 89 de 18 de junio. Ley 5/2009, de 30 de junio, de servicios sociales de Aragón B.O.A. núm.: 132 de 10 de julio. Ley 7/2009, de 22 de diciembre, de Servicios Sociales de la Rioja B.O.R. .de 28 de diciembre. Ley 14/2010, de 16 de diciembre, de servicios sociales de Castilla-La Mancha B.O.E. núm.: 38 de 14 de febrero de 2011. Ley 16/2010, de 20 de diciembre, de servicios sociales de Castilla y León - B.O.E. núm.: 7 de 8 de enero de 2011. BOCYL. núm.: 244 de 21 de diciembre de 2010 y corrección de errores en BOCYL, núm.: 23 de 3 de febrero de 2011.

5. Modificada por Real Decreto-ley 20/2012, de 30 de diciembre, de medidas urgentes en materia presupuestaria, tributaria y financiera para la corrección del déficit público B.O.E. núm.: de 31 de diciembre de 2011, Real Decreto-ley 20/2012, de 13 de julio, de medidas para garantizar la estabilidad presupuestaria y de fomento de la competitividad - B.O.E. núm.: de 14 de julio de 2012 y la Ley de Presupuestos Generales del Estado.

6. Concretamente en el ámbito de: efectividad del derecho a las prestaciones, régimen de incompatibilidad de las mismas, calendario de protección de grado, reconocimiento de grado, intensidad de protección de los servicios del catálogo, cuantías, retroactividad en la prestación, régimen de los convenios especiales en el sistema de la Seguridad Social de los cuidadores no profesionales, asignación presupuestaria de la Administración General del Estado, entre otras.

7. Modificado por Ley 57/2003, de 16 de diciembre de medidas para la modernización del gobierno local. 
y 26.1.c), regulando la creación de Comarcas u otras entidades que agrupen varios Municipios para la prestación de servicios.

El proyecto de ley de Racionalización y Sostenibilidad de la Administración Local a pesar del enorme rechazo del arco parlamentario político, así como de múltiples y diversos agentes sociales, económicos y sindicales, superó el 18 de octubre de 2013 las enmiendas de devolución al Gobierno continuando así su tramitación de manera ordinaria. En este proyecto de ley se modifica la denominación de «servicios sociales» por «evaluación e información de situaciones de necesidad social y la atención inmediata a personas en situación o riesgo de exclusión social» ( $\operatorname{art}^{\circ}$. 25.2 e del Anteproyecto de Ley), omitiéndose la prevención y la promoción.

La implantación del Plan Concertado para el Desarrollo de Prestaciones Básicas de Servicios Sociales de Corporaciones Locales en 1988 contribuyó a la ampliación de las prestaciones de los servicios sociales municipales y a la progresiva universalización de los servicios sociales de atención primaria en España (Gutiérrez, 2001, Las Heras, 2002, Subirats, 2007, Roldan et. al., 2006, Alemán, 2010) tanto desde una perspectiva territorial municipal como de personas usuarias atendidas (en 2011 se atendieron a 6.930 .978 personas, lo que supone un $15.8 \%$ de la población española), aunque en la actualidad observamos una demolición/degradación del mismo, especialmente desde 2012, atendiendo a su progresiva reducción presupuestaria, la cual para el año 2013 se sitúa en $27.593 .000 €$ representando un $2 \%{ }^{8}$ respecto de la financiación que precisa el sistema.

\section{Material y métodos}

La investigación, cuyos resultados son objeto del presente artículo, analiza de manera sistemática la evolución, estructura y dinámica del sistema de servicios sociales de atención primaria en un municipio rural situado en la Comarca Oriental de la Región de Murcia desde una perspectiva longitudinal (1996-2011), transversal (2012) y de tendencias, contrastando sus resultados con investigaciones similares en el ámbito regional y nacional.

Las unidades de análisis han sido el universo total de ciudadanos del municipio de Beniel que han sido sujetos de atención por parte de los servicios sociales municipales en el periodo comprendido entre 1996 y 2011 y que asciende a 4.562 personas distintas Este dato representa al 40,7 \% de la

8. Supone una disminución de un $58.34 \%$ respecto de 2012 y de un $68 \%$ respecto de 2011 (ver informes recientes en Asociación Estatal de Directoras y Gerentes de Servicios Sociales. http://www.directoressociales.com/index.php Consulta 15/09/2013) 
población actual del municipio, el cual cuenta con 11.198 habitantes (INE 2013) ${ }^{9}$. El conjunto de personas atendidas pertenecen a un total de 3.325 unidades familiares. En la investigación se han analizado las necesidades que han presentado las personas usuarias durante dicho período mencionado, lo que ha supuesto el análisis de 6.035 informes diagnósticos y 8.894 intervenciones sociales profesionales. Por consiguiente, la investigación se vincula con la totalidad del censo, siendo las unidades de análisis tanto las personas usuarias como las unidades familiares, las atenciones recibidas y las respuestas ofrecidas por los servicios sociales municipales a las mismas.

Tabla 1.Distribución relacional de las dimensiones objeto de estudio y sujetos

\begin{tabular}{|l|c|c|c|c|c|}
\hline \multirow{2}{*}{ DIMENSIONES } & \multicolumn{5}{|c|}{ PERSONAS USUARIAS } \\
\cline { 2 - 6 } & $1996-1999$ & $2000-2003$ & $2004-2007$ & $2008-2011$ & TOTAL \\
\hline USUARIOS & $683(15 \%)$ & $814(18 \%)$ & $970(21 \%)$ & $\begin{array}{c}2095 \\
(46 \%)\end{array}$ & $\begin{array}{c}4562 \\
(100 \%)\end{array}$ \\
\hline NECESIDADES & $850(14 \%)$ & $1075(18 \%)$ & $\begin{array}{c}1306 \\
(22 \%)\end{array}$ & $\begin{array}{c}2804 \\
(46 \%)\end{array}$ & $\begin{array}{c}6035 \\
(100 \%)\end{array}$ \\
\hline RECURSOS & $1066(12 \%)$ & $1295(14 \%)$ & $\begin{array}{c}1572 \\
(18 \%)\end{array}$ & $\begin{array}{c}4961 \\
(56 \%)\end{array}$ & $\begin{array}{c}8894 \\
(100 \%)\end{array}$ \\
\hline
\end{tabular}

Fuente: Elaboración propia.

La investigación parte de la hipótesis de que el perfil y necesidades de las personas usuarias y los recursos aplicados a éstas por parte de la estructura básica de los servicios sociales municipales durante los últimos dieciséis años se ha ido modificando en razón del contexto social e institucional, el desarrollo normativo de las políticas sociales y la crisis. Tiene, por tanto, una doble finalidad, conocer la evolución y tendencias del perfil y necesidades de las personas usuarias y analizar las respuestas «dinámicas» institucionales ofrecidas a éstas desde los servicios sociales municipales. Ambos objetivos son vertientes inseparables de una misma línea discursiva y de investigación

9. El municipio de Beniel se encuentra situado al este de la región y forma parte de la denominada Comarca Oriental de la Región de Murcia, cuenta con 11.198 habitantes y una densidad de 1.108,7 habitantes/ $\mathrm{km}^{2}$ (INE 2013). Los servicios sociales se implantaron en 1989, aunque es en 1991 cuando se integra en el Centro de Servicios Sociales de la Comarca citada, la cual desarrolla su acción en cuatro municipios con una población cercana a los 30.000 habitantes. 
analítica al encontrarse dialécticamente relacionadas con los contextos sociales, estructurales e institucionales de cada momento.

Los objetivos especificos que orientaron la investigación, son los siguientes:

1. Caracterizar la evolución del perfil socio demográfico de las personas usuarias.

2. Analizar la evolución de las necesidades sociales de las personas usuarias y de los recursos aplicados.

3. Describir el perfil, necesidades y recursos actuales y su proyección futura en el contexto local de estudio.

Con el fin de dotar a la investigación de un marco ordenado de acercamiento a la realidad objeto de estudio se identificaron como dimensiones de análisis, las siguientes:

- Perfil de los usuarios de los servicios sociales de atención primaria: sexo; edad; nacionalidad y zona de residencia.

- Evolución de las necesidades sociales de las personas usuarias: número - evolución cuatrienal - distribución por tipologías a partir de identificación necesidad principal dictaminada.

- Evolución de los recursos aplicados: número de usuarios que se han beneficiado de prestaciones - evolución cuatrienal - distribución por tipologías.

- Situación actual y percepciones desde sus actores.

- Tendencias percibidas de los servicios sociales desde los actores estratégicos del municipio.

Considerando la amplitud y complejidad del fenómeno a estudiar, se han empleado diversas técnicas de recogida de datos con el fin de incrementar la eficiencia de las mismas, concretamente la ficha social al universo de unidades de análisis y la entrevista en profundidad a informantes estratégicos para el análisis transversal y de proyección/tendencias.

Se ha considerado la ficha social como el instrumento más idóneo como fuente para la recogida de datos al encontrarse validado y disponer de la información fehaciente de todas y cada una de las unidades del universo de análisis, dimensiones y variables objeto de estudio. Ofrece datos uniformes, homogéneos y verificados, por lo que su uso ha permitido cumplir los objetivos de conocimiento con la máxima fiabilidad y validez científica, al utilizar toda la información de dicha fuente y coincidir su objeto con el propio de la presente investigación. Considerando la amplitud temporal (16 años) se ha 
considerado oportuno agrupar los datos por estratos cuatrienales (96-99 / 0003 / 04-07 / 08-11) con el fin de que cada intervalo tenga la misma longitud temporal, siendo, además, una decisión que se vincula con cortes temporales donde acontecen cambios sustanciales en el contexto social y normativo que afecta a las políticas sociales (Ley de Extranjería, Ley de Dependencia, la crisis económica) y, por tanto, vinculado a la hipótesis de estudio.

Por otra parte, las entrevistas en profundidad se han orientado a conocer los aspectos más valorativos y dinámicos tales como la situación actual y tendencias de los servicios sociales (perfil necesidades y recursos) desde los actores estratégicos en dicha área en el municipio. El universo de actores estratégicos vinculados directamente con el área específica de los servicios sociales del municipio ascendía a 13. Con el fin de garantizar la inclusión en la muestra de los diversos elementos de la población (políticos, técnicos y asociativos) en proporciones lo más cercanas posibles a la realidad, el diseño muestral adoptado ha sido el estratégico por cuotas/estratificado atendiendo a las diferentes categorías/características del universo. Concretamente se entrevistó al único responsable político, a 5 técnicos representativos de todas las áreas (servicios sociales generales, especializados e inserción laboral) y 3 asociaciones representativas del sector de iniciativa social (atención general, mujer y personas mayores).

Para el análisis de los datos se utilizó el análisis multivariable y técnicas cuantitativas y comparativas estadísticas descriptivas tales como: tablas de distribución de frecuencias y porcentajes y medidas de tendencia central y de dispersión. Para el análisis de la información reunida por entrevistas en profundidad se utilizaron técnicas cualitativas que han permitido un análisis de contenido mediante la identificación de similitudes y diferencias entre los grupos de interés (entre todos y por categorías).

\section{Resultados}

Durante el periodo comprendido entre 1996-2011 se atendieron a 4.562 personas (distintas) representando al 40,7\% del total de habitantes del municipio (considerando para el ello el dato de población de 2013). Las personas usuarias pertenecen a 3.325 unidades familiares, lo que representa un ratio de 1.4 persona usuaria/familia atendida, mostrándose constante su media en todo el periodo de estudio. El análisis longitudinal nos muestra una desigual distribución temporal destacando que el $46 \%$ de las personas atendidas entre 1996-2011 han sido usuarias entre 2008-2011, incrementándose en 22 puntos respecto del periodo inmediatamente anterior 2004-2007. En igual sentido sucede con las unidades familiares con el $44 \%$ (diferencial del $22 \%$ 
respecto del anterior período). Por otra parte, señalar que en 2011 el número de personas usuarias atendidas ascendió a 961 y conforman un total de 708 unidades familiares, representando al $21 \%$ del total de personas usuarias y unidades familiares atendidas durante el período 1996-2011. Pero si lo relacionamos con respecto al último período comprendido entre 2008-2011 representaría el $46 \%$ y $48 \%$, respectivamente. De esta manera podemos afirmar que el año 2011 representa casi el 50\% de dicho período (4 años) lo que constata la intensidad de personas usuarias y familias atendidas y, por consiguiente, el elevado número de personas en situación de necesidad que empiezan a detectarse a partir de 2011.

Gráfica 1. Distribución de unidades familiares y personas usuarias de Servicios Sociales en el periodo $1996-2011$

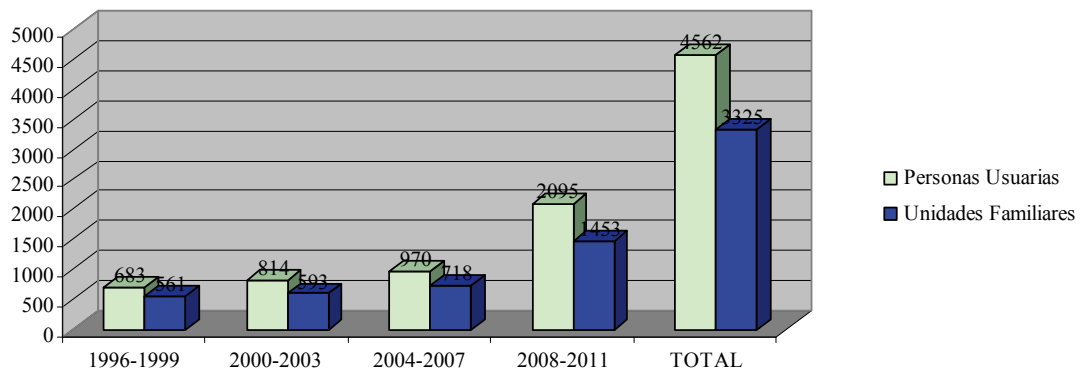

Fuente: Elaboración propia.

\subsection{Perfil de las personas y familias}

El $61 \%$ de las personas atendidas son mujeres y el 39\% son varones; una distribución constante en el periodo de estudio (1996-2011) a excepción del intervalo 2008-2011 donde se observa una reducción del 5\% de mujeres mostrando así un cambio de tendencia en el perfil de género de las personas usuarias de los servicios sociales, aunque muy incipiente. Esta tendencia se encuentra en consonancia con la información procedente de las entrevistas en profundidad que nos informan que es la mujer el sexo predominante como persona usuaria en el presente y en el futuro, pero a su vez existe la percepción de que con mayor frecuencia los hombres se van incorporando como personas usuarias al sistema.

«Mayoritariamente, y en un primer momento, suelen ser mujeres, [...], las que acuden [...], bien con una demanda propia, o de algún miembro de la familia $[\ldots] »(\mathrm{E} 9)$ 
«[... nos encontramos, aunque en menor grado, hombres [...] que han perdido su empleo.» (E7)

En cuanto a la edad el $26 \%$ de las personas beneficiarias se encuentran entre 30-44 años, el 18\% tiene 75 o más años; el 15\% son menores de 14 años o entre 45-59 (cada intervalo); el 14\% entre $15-29$ y el $12 \%$ entre $60-74$. Relacionando este dato con la estratificación de los habitantes del municipio, encontramos similitudes en torno a los grupos de edad, con la excepción de 75 o más años que representa en las personas usuarios el 18\% mientras que supone el $6 \%$ de la población total. Esta dispersión viene determinada porque dicho grupo de edad feminizado se encuentra especialmente vulnerable $y$, por tanto, necesitado de las prestaciones que ofrece el sistema de servicios sociales. Los informantes clave señalan que son las personas en edad adulta (en los tramos comprendidos entre 30 y 60 años) y los jóvenes, los grupos que preferentemente son personas usuarias en la actualidad y en el futuro, debido a las peculiares circunstancias que les afectan. Destaca la coincidencia de los informantes respecto de la percepción de una mayor presencia de los jóvenes como personas usuarias. Este dato es relevante, en tanto en cuanto, es un sector «invisible» en los servicios sociales y que a pesar de ser un colectivo en situaciones de necesidad (altos índices de desempleo y/o precariedad laboral, quiebra económica, etc.) objeto del sistema no acuden al mismo en la misma intensidad.

«Ha aumentado el número de jóvenes que necesitan ayuda.» (E2)

«Además creo que acudirán más jóvenes [...] sin formación académica ni cualificación profesional.» (E8)

En cuanto a la nacionalidad se constata a partir de 2004 que las personas usuarias de nacionalidad española descienden y se incrementa de manera exponencial (cuadruplicado) la población procedente de Marruecos, Ecuador y Ucrania. Pero este dato es relativo, dado que al analizarlo de manera comparativa con el total de población española y extranjera residente en el municipio atendiendo al último Padrón Municipal de Habitantes, se constata que se está atendiendo al 38\% de la población con nacionalidad española y al 37\% de la población con otras nacionalidades, lo que nos lleva a afirmar que el incremento se encuentra directamente relacionado con el universo de residentes y su normalización en el acceso a los servicios sociales.

Por su parte, los informantes clave señalan que son las personas inmigrantes las que fundamentalmente acceden y usan de los servicios sociales municipales tanto en la actualidad como en el futuro.

«Lo que pienso es que son [...] extranjeras [...].» (E3) 
«La población inmigrante tiene una presencia importante.»(E6)

«La diferencia será que vendrán más españolas si sigue la cosa como va.» (E5)

Esta percepción contrasta con los datos objetivos de personas usuarias atendidas al seguir siendo predominante las personas de nacionalidad española frente a las extranjeras.

Como conclusión del perfil de las personas usuarias, las entrevistas en profundidad muestran la coincidencia mayoritaria de que si se mantiene el contexto actual de recesión económica junto a los cambios estructurales previstos desde las distintas administraciones que llevan implícito un descenso en el gasto social se va a producir un cambio en el número y, por tanto, en el perfil de las personas usuarias del sistema, lo que se ha venido a denominar la «democratización de la pobreza».

"Creo que personas que hoy no están en la cola de los servicios sociales tendrán que ir. No se le ve salida a la crisis. [...] Va a haber más pobres.» (E3)

"[...], pero si la situación actual no mejora, es posible que los sectores de población atendidos se amplien a personas y familias que hasta el momento no han sido usuarios de servicios sociales.» (E6)

"Creo que personas que hoy no están en la cola de servicios sociales tendrán que ir.» (E3)

\subsection{Demandas y necesidades sociales}

En el periodo de estudio (1996-2011) el número de personas usuarias que han precisado de un diagnóstico profesional ante situaciones de necesidad ha ascendido a 6.035 (1,32 diagnóstico/usuario/a). Se constata que entre 20082011 se produce la mayor concentración de las necesidades $(46,5 \%)$ de todo el período.

Durante todo el período el 33\% de las personas usuarias atendidas han presentado una necesidad principal relacionada con el sector de referencia de personas mayores, seguido del 30\% de personas con discapacidad, el 14\% familia, el 10\% inmigrantes y el 5\% mujer. Del análisis longitudinal comprobamos que el sector de personas con discapacidad se incrementa significativamente en el período 2000-2003, el de personas mayores entre 20042007 y el de inmigrantes 2008-2011, situándose este último en tercer lugar en 2008-2011, inmediatamente después de las personas con discapacidad. Este especial incremento de las necesidades vinculadas con la migración marca una tendencia en sintonía con el perfil analizado anteriormente. Asimismo constatar que el incremento de necesidades vinculadas con las personas con discapacidad en 2000-2003 coincide con la implantación, entre otras, de la 
Tabla 2. Distribución de personas usuarias según sector de referencia en el periodo $1996-2011$

\begin{tabular}{|c|c|c|c|c|c|}
\hline \multirow{2}{*}{$\begin{array}{l}\text { SECTOR DE } \\
\text { REFERENCIA }\end{array}$} & \multicolumn{5}{|c|}{ PERSONAS USUARIAS } \\
\hline & 1996-1999 & $2000-2003$ & 2004-2007 & 2008-2011 & TOTAL \\
\hline Familia & $159(23,4 \%)$ & $133(16,3 \%)$ & $90(9,3 \%)$ & $253(12,1 \%)$ & $635(13,9 \%)$ \\
\hline Infancia & $19(2,8 \%)$ & $92(11,3 \%)$ & $64(6,6 \%)$ & $30(1,4 \%)$ & $205(4,5 \%)$ \\
\hline Juventud & $0(0,0 \%)$ & $5(0,6 \%)$ & $1(0,1 \%)$ & $14(0,7 \%)$ & $20(0,4 \%)$ \\
\hline Mujer & $13(1,9 \%)$ & $39(4,8 \%)$ & $31(3,2 \%)$ & $134(6,4 \%)$ & $217(4,8 \%)$ \\
\hline Personas mayores & $194(28,5 \%)$ & $168(20,6 \%)$ & $411(42,4 \%)$ & $725(34,6 \%)$ & $1498(32,8 \%)$ \\
\hline Personas discapacidad & $241(35,3 \%)$ & $341(41,9 \%)$ & $338(34,8 \%)$ & $466(22,3 \%)$ & $1386(30,4 \%)$ \\
\hline $\begin{array}{l}\text { Reclusos y ex- } \\
\text { reclusos }\end{array}$ & $1(0,1 \%)$ & $1(0,1 \%)$ & $1(0,1 \%)$ & $2(0,1 \%)$ & $5(0,1 \%)$ \\
\hline Minorías étnicas & $2(0,3 \%)$ & $0(0,0 \%)$ & $1(0,1 \%)$ & $1(0,0 \%)$ & $4(0,1 \%)$ \\
\hline $\begin{array}{l}\text { Marginados/ } \\
\text { transeúntes }\end{array}$ & $26(3,8 \%)$ & $15(1,9 \%)$ & $0(0,0 \%)$ & $0(0,0 \%)$ & $41(1,0 \%)$ \\
\hline Toxicómanos & $7(1,0 \%)$ & $10(1,2 \%)$ & $6(0,6 \%)$ & $4(0,2 \%)$ & $27(0,6 \%)$ \\
\hline Refugiados y asilados & $1(0,1 \%)$ & $0(0,0 \%)$ & $0(0,0 \%)$ & $0(0,0 \%)$ & $1(0,0 \%)$ \\
\hline Emigrantes & $1(0,1 \%)$ & $1(0,1 \%)$ & $1(0,1 \%)$ & $1(0,0 \%)$ & $4(0,1 \%)$ \\
\hline Enfermos mentales & $1(0,1 \%)$ & $1(0,1 \%)$ & $0(0,0 \%)$ & $0(0,0 \%)$ & $2(0,0 \%)$ \\
\hline Enfermos terminales & $1(0,1 \%)$ & $2(0,3 \%)$ & $1(0,1 \%)$ & $0(0,0 \%)$ & $4(0,1 \%)$ \\
\hline Otros & $17(2,5 \%)$ & $4(0,5 \%)$ & $1(0,1 \%)$ & $11(0,5 \%)$ & $33(0,7 \%)$ \\
\hline Inmigrantes & $0(0,0 \%)$ & $2(0,3 \%)$ & $24(2,5 \%)$ & $454(21,7 \%)$ & $480(10,5 \%)$ \\
\hline TOTAL & $683(100 \%)$ & $814(100 \%)$ & $970(100 \%)$ & $2095(100 \%)$ & $4562(100 \%)$ \\
\hline
\end{tabular}

Fuente: Elaboración propia.

prestación familiar por hijo a cargo y el incremento relacionado con personas mayores en el período 2004-2007 (duplicado) está relacionado con la implantación de la Ley 39/2006.

En cuanto a tendencias, la información obtenida de las entrevistas en profundidad es la relativa a la incorporación al sistema de nuevas personas usuarias en situación de necesidad social que precisen por primera vez de una intervención profesional de los servicios sociales, procedentes de los sectores de población de personas mayores, jóvenes e inmigrantes.

«[...] se incorporarán como colectivo los pensionistas que cobran pensión y que por la situación del pago de medicamentos y de las subidas, en general, necesitarán ayuda económica de los servicios sociales.» (E3)

«[...] en los próximos dos años los sectores de población que se verán más afectados serán juventud, debido a la dificultad para encontrar un primer empleo, así como los inmigrantes [...] algunos trabajan sin contrato y no podrán pedir prestación por desempleo [...].» (E4) 
Atendiendo a la categorización de necesidades el 68\% de las personas usuarias atendidas han presentado una necesidad relacionada con una adecuada información sobre el acceso a recursos, el 17\% con una adecuada convivencia personal, el $9 \%$ falta de medios para la cobertura de las necesidades básicas y el $6 \%$ restante con integración social.

Tabla 3. Distribución de personas usuarias según necesidades sociales en el periodo $1996-2011$

\begin{tabular}{|c|c|c|c|c|c|}
\hline \multirow[t]{2}{*}{ NECESIDADES } & \multicolumn{5}{|c|}{ PERSONAS USUARIAS } \\
\hline & 1996-1999 & $2000-2003$ & $2004-2007$ & $2008-2011$ & TOTAL \\
\hline $\begin{array}{l}\text { Información sobre el } \\
\text { acceso a recursos }\end{array}$ & $678(79,8 \%)$ & $767(71,3 \%)$ & $742(56,8 \%)$ & $1894(67,5 \%)$ & $4081(67,6 \%)$ \\
\hline $\begin{array}{l}\text { Adecuada } \\
\text { convivencia } \\
\text { personal / familiar }\end{array}$ & $89(10,5 \%)$ & $185(17,2 \%)$ & $393(30,1 \%)$ & $345(12,3 \%)$ & $1012(16,8 \%)$ \\
\hline Integración social & $58(6,8 \%)$ & $45(4,2 \%)$ & $46(3,5 \%)$ & $212(7,6 \%)$ & $361(6,0 \%)$ \\
\hline $\begin{array}{l}\text { Falta de medios para } \\
\text { cubrir nec. básicas }\end{array}$ & $25(2,9 \%)$ & $78(7,3 \%)$ & $125(9,6 \%)$ & $353(12,6 \%)$ & $581(9,6 \%)$ \\
\hline TOTAL & $850(100 \%)$ & $1075(100 \%)$ & $1306(100 \%)$ & $2804(100 \%)$ & $6035(100 \%)$ \\
\hline
\end{tabular}

Fuente: Elaboración propia.

Tal y como se constata las necesidades relacionadas con la falta de medios para la cobertura de las necesidades básicas y de integración social son las que se sitúan por encima de la media en el período comprendido entre 2008-2011, en consonancia con las dificultades económicas que atraviesan las personas y familias a raíz de la crisis económica y financiera y que precisan de la atención de los servicios sociales. Este incremento en dichas necesidades se relaciona, a su vez, con el incremento de las situaciones de desempleo, quiebra económica e incremento de la exclusión social (Pastor, 2013), entre otros.

En cuanto a la evolución de las necesidades sociales de manera más específica el 54\% de las personas usuarias atendidas han presentado una necesidad relacionada con información sobre el acceso a recursos del sistema de servicios sociales, el 14\% con otros sistemas, el 10\% con una adecuada convivencia personal/familiar motivada por desajustes convivenciales; el 9,4\% ante la falta de medios para la cobertura de las necesidades básicas y el 7\% por limitación de autonomía personal y/o situaciones de dependencia.

Respecto a las necesidades específicas se vuelve a constatar que son las necesidades relacionadas con la falta de medios para la cobertura de las necesidades básicas y de integración social, laboral y escolar las que se sitúan por encima de la media en el período 2008-2011, destacando, de un análisis 
más desagregado, que las necesidades de integración escolar se incrementan especialmente a partir de 2004 motivado, entre otros, por las reagrupaciones familiares.

Tabla 4. Distribución personas usuarias según necesidades sociales específicas en el periodo $1996-2011$.

\begin{tabular}{|c|c|c|c|c|c|}
\hline \multirow[t]{2}{*}{ NECESIDADES } & \multicolumn{5}{|c|}{ PERSONAS USUARIAS } \\
\hline & 1996-1999 & $2000-2003$ & 2004-2007 & $2008-2011$ & TOTAL \\
\hline $\begin{array}{l}\text { Información para } \\
\text { acceso al Sistema de } \\
\text { SS. SS. }\end{array}$ & $455(53,6 \%)$ & $579(53,9 \%)$ & $639(48,9 \%)$ & $1576(56,2 \%)$ & $3249(53,8 \%)$ \\
\hline $\begin{array}{l}\text { Información otros } \\
\text { Sist. Protección } \\
\text { Social }\end{array}$ & $223(26,2 \%)$ & $188(17,5 \%)$ & $103(7,9 \%)$ & $318(11,3 \%)$ & $832(13.8 \%)$ \\
\hline $\begin{array}{l}\text { Adecuada } \\
\text { convivencia } \\
\text { familiar: desajustes }\end{array}$ & $47(5,5 \%)$ & $98(9,1 \%)$ & $187(14,3 \%)$ & $245(8,8 \%)$ & $577(9,6 \%)$ \\
\hline $\begin{array}{l}\text { Adecuada } \\
\text { convivencia: } \\
\text { (limitación } \\
\text { autonomía personal/ } \\
\text { dependencia)) }\end{array}$ & $42(5,0 \%)$ & $878,1 \%)$ & $206(15,8 \%)$ & $100(3,5 \%)$ & $435(7,2 \%)$ \\
\hline Integración social & $52(6,1 \%)$ & $33(3,1 \%)$ & $20(1,6 \%)$ & $159(5,7 \%)$ & $264(4,4 \%)$ \\
\hline Integración laboral & $4(0,5 \%)$ & $4(0,3 \%)$ & $7(0,5 \%)$ & $21(0,8 \%)$ & $36(0,6 \%)$ \\
\hline Integración escolar & $2(0,2 \%)$ & $8(0,8 \%)$ & $19(1,5 \%)$ & $32(1,1 \%)$ & $61(1,0 \%)$ \\
\hline $\begin{array}{l}\text { Falta de medios } \\
\text { para cobertura nec. } \\
\text { básicas }\end{array}$ & $23(2,7 \%)$ & $71(6,6 \%)$ & $123(9,4 \%)$ & $353(12,6 \%)$ & $570(9,4 \%)$ \\
\hline $\begin{array}{l}\text { Falta de medios por } \\
\text { emergencias }\end{array}$ & $2(0,2 \%)$ & $7(0,6 \%)$ & $2(0,1 \%)$ & $0(0,0 \%)$ & $11(0,2 \%)$ \\
\hline TOTAL & $850(100 \%)$ & $1075(100 \%)$ & $1306(100 \%)$ & $2804(100 \%)$ & $6035(100 \%)$ \\
\hline
\end{tabular}

Fuente: Elaboración propia.

Los informantes clave identifican como principales las necesidades relacionadas con: falta de recursos propios para cobertura de necesidades básicas (fundamentalmente recursos económicos), adecuada convivencia personal/ familiar (principalmente situaciones de dependencia) e integración (fundamentalmente laboral).

«En la actualidad, las familias y la población en general del municipio están teniendo serias dificultades para la cobertura de necesidades básicas de sus miembros y, sobre todo, de los menores.» (E7) 
«En general, la mayor parte de las personas que acuden a servicios sociales demandan ayudas económicas, dada la situación actual y poder hablar con la profesional de su situación.» (E6)

\subsection{Beneficios / recursos / sistema de respuestas a las necesidades sociales}

Durante el periodo de referencia del estudio se ha aplicado un total de 8.894 recursos/prestaciones, constatándose que en el intervalo 2008-2011 se concentra la aplicación de los recursos (56\%) de todo el período, ascendiendo a un ratio de 2.4 recurso aplicado/usuario/a. Al analizar el año 2011 se constata que la proporción aplicada de recurso/usuario se reduce en 0.6 puntos de la media en el periodo 2008-2011 (1.75), lo que confirma una reducción de los recursos disponibles / aplicados en el sistema de servicios sociales en sintonía con el progresivo recorte en los servicios sociales en España. En igual sentido se manifiestan a su vez los informantes clave.

«[...] tenemos serias dificultades para articular recursos sociales que palien esas carencias y dificultades, sobre todo si nos referimos a prestaciones de tipo económico.» (E7)

"[...] ha habido recortes importantes en materia de recursos sociales.» (E1)

«Supongo que las situaciones de crisis [...] harán que se produzca una sobrecarga en el sistema de servicios sociales, habiendo una mayor demanda [... y si se mantiene la tónica general de un recorte en servicios, se acabará dejando a la UTS como gestora de recursos sin posibilidad real de intervención.» (E9)

«La situación actual no da garantías de mantener las coberturas actuales» (E6)

Como se muestra en la siguiente tabla (5), en el periodo 1996-2011, el 40,3 $\%$ de los recursos aplicados son prestaciones de información, orientación, valoración y movilización; el 26,1 \% de apoyo a la unidad convivencial y ayuda a domicilio, el $19,4 \%$ son recursos complementarios para la cobertura de necesidades básicas y el 13,5\% de prevención e inserción social. Destacar que las prestaciones relacionadas con el alojamiento alternativo en todo el período no alcanzan el $1 \%$.

Las prestaciones de prevención e inserción social y de apoyo a la unidad convivencial son las que se sitúan por encima de la media en el periodo 2008-2011, llegando a duplicarse en el primer caso. Contrasta la disminución de casi un $50 \%$ entre la media del período total y la del comprendido entre 2008-2011 de las prestaciones relacionadas con la cobertura de necesidades básicas cuando anteriormente hemos constatado que dichas necesidades han aumentado en ese mismo periodo en un tercio. De esta manera podemos afirmar que las necesidades de cobertura de necesidades básicas 
Tabla 5. Distribución personas usuarias según prestaciones básicas en periodo 1996- 2011

\begin{tabular}{|l|r|r|r|r|r|}
\hline \multirow{2}{*}{ PRESTACIONES } & \multicolumn{5}{|c|}{ PERSONAS USUARIAS } \\
\cline { 2 - 6 } & $1996-1999$ & $2000-2003$ & $2004-2007$ & \multicolumn{1}{|c|}{$2008-2011$} & \multicolumn{1}{c|}{ TOTAL } \\
\hline $\begin{array}{l}\text { Información/ } \\
\text { orientación/ } \\
\text { valoración/ } \\
\text { movilización. }\end{array}$ & $560(52,5 \%)$ & $509(39,3 \%)$ & $633(40,3 \%)$ & $1883(38,0 \%)$ & $3585(40,3 \%)$ \\
\hline $\begin{array}{l}\text { Apoyo a unidad } \\
\text { convivencial / ayuda } \\
\text { a dom. }\end{array}$ & $105(9,8 \%)$ & $298(23,0 \%)$ & $548(34,9 \%)$ & $1371(27,6 \%)$ & $2322(26,1 \%)$ \\
\hline $\begin{array}{l}\text { Actuaciones y } \\
\text { medidas alojamiento } \\
\text { alternativo }\end{array}$ & $6(0,6 \%)$ & $13(1,0 \%)$ & $29(1,8 \%)$ & $11(0,2 \%)$ & $59(0,7 \%)$ \\
\hline $\begin{array}{l}\text { Prevención e } \\
\text { inserción social }\end{array}$ & $8(0,8 \%)$ & $12(0,9 \%)$ & $15(0,9 \%)$ & $1164(23,5 \%)$ & $1199(13,5 \%)$ \\
\hline $\begin{array}{l}\text { Recursos compl. } \\
\text { para cobertura nec. } \\
\text { básicas }\end{array}$ & $387(36,3 \%)$ & $463(35,8 \%)$ & $347(22,1 \%)$ & $532(10,7 \%)$ & $1729(19,4 \%)$ \\
\hline TOTAL & $1066(100 \%)$ & $1295(100 \%)$ & $1572(100 \%)$ & $4961(100 \%)$ & $8894(100 \%)$ \\
\hline
\end{tabular}

Fuente: Elaboración propia.

no encuentran una respuesta en cuanto a aplicación de recursos vinculados a dichas necesidades.

De un análisis más desagregado y en cuanto a las prestaciones de información, orientación, valoración y movilización se refiere, el 63\% de las personas usuarias han sido beneficiarias de la prestación de información y orientación de recursos del sistema de servicios sociales y el 37\% de otros sistemas de protección. Así mismo, en el análisis longitudinal se comprueba que mientras la información y orientación sobre reconocimiento del grado de discapacidad se incrementa a lo largo del período, la información sobre prestaciones económicas de la Comunidad Autónoma y de pensiones no contributivas sufre un progresivo descenso desde 2004.

Por otra parte, a lo largo de todo el periodo, el 26,1\% de las personas usuarias del sistema han sido beneficiarias de la prestación de apoyo a la unidad convivencial y ayuda a domicilio, aunque la entrada en vigor de la Ley 39/2006 incide directamente en los recursos que contiene esta prestación. Cabe destacar que del análisis longitudinal se constata un incremento de las actuaciones de inserción social a partir de 2011, con lo que se constata, de nuevo, el incremento de situaciones de dificultad social que precisan de la valoración e intervención profesional orientada a la integración/inserción social, 
situación en sintonía con el contexto actual de exclusión social como muestran diferentes estudios (Amnistía Internacional, 2011; Laparra y Pérez, 2011; García y Ramírez, 2012, FOESSA, 2013, García, Barriga, Ramírez y Santos, 2013). En igual sentido se manifiestan los informantes clave, que destacan como importantes ahora y en el futuro los recursos destinados a la inserción laboral y prevén un incremento en las personas beneficiarias de los mismos.

«Debido a la falta de empleo las personas necesitarán más recursos a nivel de inserción y formación laboral.» (E4)

\section{Discusión}

Los resultados del trabajo empírico realizado constatan la hipótesis que ha orientado la investigación, concretamente, que la evolución y tendencias del perfil y necesidades de las personas usuarias y las respuestas institucionales actuales y futuras de la estructura básica de los servicios sociales en el municipio se han ido modificando en razón del contexto social e institucional y del desarrollo normativo de las políticas sociales vinculadas a los servicios sociales, con un claro impacto de la crisis y los recortes en el sistema y las personas.

La investigación muestra que el número de personas que precisan de la atención y prestaciones de los servicios sociales ha ido ascendiendo progresivamente, constatándose un incremento exponencial a partir de la crisis económica. Así solo en un año, concretamente en el 2011 se atendió a un 21\% del total de personas usuarias atendidas en 16 años y al 50\% de los últimos 4 años. Este dato es representativo de la repercusión que ha tenido la crisis en la ciudadanía del municipio, pero esta realidad no es particular, todo lo contrario, se trata de una situación que está sucediendo en los servicios sociales en el contexto nacional conforme a los estudios específicos sobre la materia (Lima, 2011; Barriga, 2012; Gallego y Subirats, 2012; Gustavo y Ramírez, 2012; Roldan y Castañer, 2012; Pastor, 2012a; Asociación de Directores y Gerentes de Servicios Sociales, 2012; Alguacil, 2012; Fundación FOESSA, 2013, García, Barriga, Ramírez y Santos, 2013). De esta manera, se constata que el incremento de personas usuarias ha aumentado a consecuencia y en consonancia temporal a la crisis económica y financiera.

El perfil de las personas usuarias se caracteriza por ser mayoritariamente mujer, de nacionalidad española, residente en el casco urbano y en edades comprendidas entre 30 y 60 años. Un perfil que lejos de ser estático se nos muestra dinámico y cambiante con una tendencia a una progresiva, aunque lenta, equiparación de género (López, 2012) en el acceso, uso y disfrute de las prestaciones sociales y que encuentra múltiples causas, entre otras, en el incremento en el número de personas inmigrantes (mayoritariamente hombres) 
usuarias y el aumento de hombres que precisan, en ambos casos, atención personalizada para la resolución de los problemas que les afecta directamente (desempleo, cobertura necesidades básicas, etc.). Además de estos factores debemos añadir que los hombres se han visto más afectados por las situaciones de desempleo que las mujeres, por lo que son ellos los que deben acudir a los servicios sociales para solicitar las prestaciones y ayudas que precisa la unidad convivencial al encontrarse la mujer trabajando. De manera más particular, las características del empleo femenino en el municipio (industria agroalimentaria) se ha visto menos afectado por la situación de crisis por lo que sus ingresos han pasado de ser subsidiarios a ser principales, siendo el hombre el que debe ocuparse de algunas tareas tradicionalmente vinculadas a las mujeres como es el de acudir a los servicios sociales. Por otra parte y como se ha señalado anteriormente, la progresiva normalización de los servicios sociales contribuye a que éstos no sean percibidos como «degradantes», «vergonzosos» o «marginales» para quien los «usa» sino como un recurso más del municipio al que cualquier ciudadano puede acudir ante situaciones concretas. Este cambio de imaginario incide en que progresivamente el hombre se convierta en persona usuaria. No debemos olvidar que, habitualmente, la persona usuaria es únicamente aquella que presenta una necesidad social y que en muchas ocasiones dicha situación de dificultad no se vincula directamente con ella individualmente, sino con la unidad familiar o incluso con algún miembro distinto del que accede como usuario/a y, en cualquier caso, siempre con efectos en la unidad convivencial.

La investigación también nos muestra que el número de necesidades presentadas ha ascendido de manera diferencial a partir de la crisis económica, donde se constata un aumento sustancial. Pero profundizando en el ámbito de las necesidades, comprobamos que la necesidad principal que motiva la intervención se ha ido modificando en el tiempo. Las personas inmigrantes, mayores y discapacitados son los sectores de referencia que han orientado mayoritariamente y de manera constante el diagnóstico y la intervención de los servicios sociales, variando en el tiempo su prelación. Los datos señalan, que las personas inmigrantes son el colectivo fundamentalmente afectado y precisado de los servicios sociales, lo que se relaciona con el actual contexto, dado que son los que están sufriendo con mayor intensidad los efectos de la crisis económica al encontrarse en situaciones previas de mayor vulnerabilidad (precariedad laboral, insuficientes apoyos familiares, déficits en hábitat, ingresos inestables, etc.) y en las fronteras de los sistemas de protección social.

En la actual situación de crisis económica y progresiva descapitalización de los sistemas de protección, las personas situadas en los márgenes se 
encuentran abocadas al abismo de la pobreza, la exclusión y la desprotección social. Esta situación se constata, a su vez, al comprobar que son las necesidades relacionadas con la falta de medios para la cobertura de las necesidades básicas y de integración social las fundamentales en la actualidad; acordes al incremento de las situaciones de desempleo, quiebra económica de las personas y exclusión social. Por otra parte, el análisis longitudinal del sector de referencia nos muestra, además, una relación directa de éste respecto de la implantación de servicios y prestaciones dirigidas a los mismos, lo que constata su relación con el desarrollo normativo.

Se constata que en el actual contexto de crisis se ha producido un progresivo descenso en la oferta de prestaciones que se ofrecen de manera efectiva a las personas usuarias. Las demandas relacionadas con prestaciones de prevención e inserción social y de cobertura de necesidades básicas son las que han sufrido un considerable incremento en la actualidad en coherencia con la actual situación de crisis. Tanto las prestaciones de carácter técnico como económico se han visto sustancialmente mermadas.

La crisis se encuentra afectando doblemente a las personas, por una parte, al encontrarse en situaciones de exclusión y, por otra, al no disponer de políticas de protección ante tales situaciones, lo que ocasiona situaciones de «abismo»y «violencia estructural». Por tanto, nos encontramos con un sistema dotado de capital humano pero infradotado en la actualidad en relación a las necesidades sociales actuales y futuras. Esta situación de descapitalización del sistema público y de aumento galopante de necesidades está ocasionando que el sistema deba acudir a la iniciativa de organizaciones no gubernamentales para conseguir que las personas puedan cubrir sus necesidades de subsistencia más básicas a través de prestaciones en especie, lo que nos vincula con escenarios de graciabilidad, asistencialismo, caridad, limosna, etc. que creíamos superados. Esta situación revela un sistema que se va debilitando muy rápidamente por los recortes del gasto público y que éstos afectan muy negativamente al desarrollo de los servicios sociales, poniendo en riesgo la sostenibilidad del sistema.

Pero esta situación no solo afecta al sistema de servicios sociales del municipio sino que se vincula a un contexto de degradación del sistema público de servicios sociales de la misma Comunidad Autónoma de la Región de Murcia. Así, los dos últimos Informes sobre Desarrollo de Servicios Sociales (García y Ramírez, 2012 y García, Barriga, Ramírez y Santos, 2013) señalan que ésta ocupa la posición número 14 (respecto de las 17 comunidades autónomas) en el desarrollo de los servicios sociales, con una calificación de «irrelevantes» y una puntuación global de 3.6 (2012) y 2.8 (2013) sobre 10. 
Entre los indicadores resaltar: falta de un catálogo de derechos, carencia de instrumentos de planificación y ordenación del sector, gasto por habitante inferior a la media nacional, etc. Esta situación de marcada debilidad del sistema regional de servicios sociales nos sitúa en una situación muy precaria en un contexto de recortes del gasto público en los sistemas de protección social en general y de los servicios sociales en particular.

Pero lejos de cifras, lo cierto es que la inadecuada/insuficiente satisfacción de las necesidades presentes redundará de manera negativa en la calidad de vida de las generaciones futuras y repercutirá en el propio desarrollo social del territorio y del sistema de servicios sociales. Analizando la aplicación de las prestaciones en el tiempo se vuelve a constatar que se encuentra estrechamente vinculado con la implantación especifica de las mismas y los sectores de población destinatarios; siendo especialmente significativo lo relacionado con el Sistema para la Autonomía y Atención a la Dependencia. Estas prestaciones han experimentado un crecimiento al mismo tiempo que han sufrido un decrecimiento las pertenecientes al propio sistema de servicios sociales. Se constata, al igual que en los estudios relativos a la aplicación de la Ley 39/2006, de Promoción de la Autonomía Personal y Atención a las personas en situación de dependencia (Rodríguez, 2011; Asociación Estatal de Directores y Gerentes de Servicios Sociales, 2012; Observatorio de la Dependencia, Ramírez, 2012) que la mayor parte de los recursos aplicados del sistema corresponden a la prestación económica de cuidados en el entorno en detrimento de los servicios de ayuda a domicilio y/o residenciales; siendo una tónica general en la aplicación de la citada ley. En este sentido, los cambios introducidos por leyes de índole presupuestaria en los dos último años (2012 y 2013) están incidiendo directamente en la mencionada Ley y está repercutiendo directamente en las economías familiares.

En resumen, los resultados de la investigación constatan la hipótesis de partida y se confirma que la evolución y tendencias del perfil y necesidades de las personas usuarias y las respuestas institucionales actuales y futuras de la estructura básica de los Servicios Sociales en el municipio se ha ido modificando en razón del contexto social e institucional y del desarrollo normativo de las políticas sociales vinculadas a los servicios sociales.

Por ello y ante la actual situación de crisis y de recortes del gasto público es preciso revitalizar el sistema mediante una promulgación de una ley de servicios sociales que otorgue derechos de ciudadanía-subjetivos; luchar por mantener e incrementar el esfuerzo presupuestario, incrementar la cobertura y cuantía de las prestaciones vinculadas con la cobertura de las necesidades básicas. En el ámbito local dichas propuestas son también de aplicación en 
su micro-desarrollo y en la propia organización más eficiente de los escasos recursos existentes.

\section{Bibliografía}

Alemán, C. (2010). Fundamentos de Servicios Sociales. Valencia: Tirant Lo Blanch AlguACIL, J. (2012). La quiebra del incompleto sistema de Servicios Sociales en España. Cuadernos de Trabajo Social, 25 (1), 63-74

Amnistía Internacional (2011). Derechos a la intemperie. Obstáculos para hacer valer los derechos económicos, sociales y culturales en España. Madrid: Amnistía Internacional

Asociación Estatal de Directores y Gerentes de Servicios Sociales (2012). Informe sobre el Estado Social de la Nación. Asociación Estatal de Directores y Gerentes de Servicios Sociales. Madrid: Roneo. (http://www.directoressociales.com/ documentos/novedades-en-discusi\%C3\%B3n.html Consultado 30/11/2012)

BARRIGA, L. (2012). La protección a las situaciones de quiebra económica de particulares. Un desafío para los Servicios Sociales en España. AZARBE Revista Internacional de Trabajo Social y Bienestar, 1, 21-38

CASAdo, D. (Coord.) (2010). Leyes de Servicios Sociales del siglo XXI. Cáritas. Fundación Fomento de Estudios Sociales y de Sociología Aplicada FOESSA.

Fundación FOESSA (2013): Desigualdad y derechos sociales. Análisis y perspectivas. Cáritas. http://www.caritas.es/noticias_tags_noticiaInfo.aspx?Id=6475

GAllEGO, R. y SubiRATS, J. (2011). Autonomies i desigualtatas a Espanya: percepcions, evolució social i politiques de benestar. (Con)textos. Barcelona: Institut d’Estudis Autonómics. Generalitat de Catalunya.

García, G. y RAmírez, J.M. (2012). Índice de desarrollo de los Servicios Sociales 2012. Asociación Estatal de Directores y Gerentes de Servicios Sociales. Madrid. Roneo. http://www.directoressociales.com/documentos/novedades-endiscusi\%C3\%B3n.html (Consultado 30/11/2012)

García, G., Barriga, L, Ramírez, J.M. y SAntos, J. (2013). Índice de desarrollo de los Servicios Sociales 2013. Asociación Estatal de Directores y Gerentes de Servicios Sociales. Madrid. Roneo. http://www.directoressociales.com/documentos/novedades-en-discusi\%C3\%B3n.html (Consultado 30/09/2013)

Gutiérrez, A. (2001). El Plan Concertado de prestaciones básicas de Servicios Sociales en España (once años del Plan Concertado). REIS, 93, 89-130

LAPARRA, M. y PÉREZ, B. (2011). El primer impacto de la crisis en la cohesión social en España. Madrid: Fundación FOESSA

Las Heras, M. P. (2002). El Sistema Público de Servicios Sociales: contribución del Trabajo Social, desafíos, oportunidades y estrategias. Revista Trabajo Social Hoy Monográfico, 9-80. 
LiMA, A. I. (2012) Trabajo social, nuevos contexto, nuevos compromisos. AZARBE Revista Internacional de Trabajo Social y Bienestar, 1, 73-86.

LÓPEZ, A. (2012). Jóvenes y Trabajo Social. Revista de Estudios de Juventud. Madrid: INJUVE. Ministerio de Sanidad, Servicios Sociales e Igualdad.

Ministerio de Sanidad, Servicios Sociales e Igualdad (2013): Catalogo de referencia de Servicios Sociales. Roneo. http://www.msc.es//ssi/familiasInfancia/ inclusionSocial/serviciosSociales/Catalogo_Servicios_Sociales/docs/Catalogo_SSSS.pdf (Consultado 30/07/2013)

PASTOR, E. (2011): Impact, effectiveness and sustainability of social policies and local democracy through the social involvement. Revista de Cercetare si Interventie Sociala (RCIS), 35, 7- 27.

- (2012a). Gobernanza de los Servicios Sociales municipales: dar voz y salida a las aspiraciones ciudadanas. Cuadernos de Trabajo Social, 25(1),143-148

- (2012b). Trabajo social, capital social, inteligencia cooperativa y diálogo significante en el ámbito local. Portularia, 12, 91-99

RAmíREZ, J. M. (2012). Informe de evaluación de la Ley de Dependencia. Una mirada a la realidad de la Región de Murcia. AZARBE Revista Internacional de Trabajo Social y Bienestar, 1, 97-109

RodríGuez, C. G. (2011). Políticas de atención a la dependencia en los regímenes de bienestar de la Unión Europea. Ponencia presentada en el Encuentro de Intervención y Políticas Sociales de Caja Madrid y X Jornadas de SIPOSO: Protección a la dependencia y leyes de servicios sociales del siglo XXI. Madrid, 3 de junio de 2011. Roneo

Roldán, E. y García, T. (2006). Políticas de Servicios Sociales. Madrid: SINTESIS

Roldán, E. y CASTAÑar, M. (2012). Servicios Sociales en tiempos de crisis. Cuadernos de Trabajo Social, 25 (1), 13-17

Subirats, J. (Dir.) (2007). Los Servicios Sociales de Atención Primaria ante el cambio social. Madrid: Ministerio de Trabajo y Asuntos Sociales

VILÀ, A. (2012). La nueva generación de leyes autonómicas de servicios sociales (2006-2011): análisis comparativo. AZARBE Revista Internacional de Trabajo Social y Bienestar, 1, 143-157. 